\title{
Investigation into the capabilities of Hall cells integrated in a non-fully depleted SOI CMOS technological process
}

\author{
Maria-Alexandra Paun*, Florin Udrea \\ High Voltage Microelectronics and Sensors (HVMS) Group \\ Department of Engineering, University of Cambridge \\ 9 JJ Thomson Avenue, Cambridge CB3 0FA, United Kingdom \\ *Corresponding author: map65@cam.ac.uk, maria_paun2003@yahoo.com
}

\begin{abstract}
The behaviour of SOI Hall cells integrated in a non-fully depleted SOI ("Silicon-On-Insulator") CMOS technology is investigated, with an emphasis on the study of their main parameters. To meet these objectives, a particular optimum structure has been designed, integrated and subsequently analyzed. The performance evaluation of this Hall cells is carried out by means of both a threedimensional physical model and measurements. The Hall voltage, electrostatic potential distribution, space charge and sensitivity have all been evaluated. In addition, the Hall mobility has been studied through simulations. In order to complete the performance assessment of the Hall cells studied, experimental results for the offset and variation of the sensitivity with the temperature are also provided.
\end{abstract}

Index Terms - Hall cells, design, SOI, three-dimensional physical model, optimization, temperature variation, offset, Hall mobility

\section{INTRODUCTION}

$\mathrm{I}^{\mathrm{n}}$ $n$ the couple of last decades, CMOS Hall Effect sensors have served in different practical and industrial applications, for the detection of current, in DC motors, for contactless measurements of position or angle, etc. They have also served a purpose in electronic compasses for direct magnetic field sensing. Their employment in the above mentioned applications is owed to their low fabrication cost, high level of integrability, versatility and robustness $[1,2]$.

Hall Effect sensors have been fabricated in both regular bulk and SOI (Silicon $\boldsymbol{O}$ n Insulator) CMOS technologies. However, the advantages of SOI Hall sensors (higher magnetic sensitivity, possibility to use lower biasing voltage, smaller leakage current through the dielectric, less noise, greater radiation resistance [3]) make them more appealing to the industry nowadays.

In [4], a high sensitivity Hall sensor fabricated on a SOI wafer using surface micromachining technique was presented. Previous works have been concerned with solid state meters based on SOI Hall sensors, for power and energy measurements [5,6]. An architecture based on SOI CMOS Hall effect sensor was used for high temperature applications [7]. SOI Hall cells behaviour was previously investigated by the author through three-dimensional physical models $[8,9]$.

As there is a great correlation between the geometry of the CMOS Hall Effect devices and their performance, various designs were proposed and their behaviour was analyzed [10]. On this occasion, extensive measurements on the offset and the offset temperature drift of regular bulk CMOS were performed. Therefore, offset values lower than $30 \mu \mathrm{T}$ at room temperature and offset temperature drifts less than $\pm 0.3 \mu \mathrm{T} /{ }^{\circ} \mathrm{C}$ were reached by the first author with specific structures [11].

The work carried out in this paper intends to look into the behaviour of Hall cells in an SOI CMOS technological process and to assess their performance, with an emphasis on a particular optimum structure. In Section II, considerations on the three-dimensional physical simulations methodology are included. Section III focuses on presenting the threedimensional physical model of Hall cells, with an emphasis on the Optimum cell, also giving simulation results on the electrostatic potential distribution and space charge. Throughout Section IV, we were concerned with extracting both simulation and measurements results on $\mathrm{I}-\mathrm{V}$ characteristic, Hall voltage, absolute sensitivity and Hall mobility. Temperature variation of the absolute sensitivity is also studied. On this occasion, the offset has been measured. The conclusions are drawn in Section V.

\section{ON The ThreE-Dimensional Physical Simulations}

In the optimization stages of the CMOS Hall devices, different geometries have been designed and integrated in both regular bulk and SOI technological processes. There has been research by the first author, in which the geometry influence on the Hall device performance has been extensively studied in [10], when different structures (L, XL, basic, borderless, etc.) have been analyzed. Electrical contacts dimension selection influence on the sensitivity was studied in [12], as well as effects of device scaling on the offset values of crosslike Hall cells in [11]. In the fabrication of the Hall devices, the technological process is important. Different CMOS technologies (doping, implantation thickness variation) have been investigated through circuit models in [13].

Three dimensional physical simulations of magnetic field influence on the semiconductors were previously performed by the author in [8,9], in order to assess the device performance.

\section{A. Carrier transport in semiconductors}

Assuming low injection, the classical carrier transport model for an n-type semiconductor is based on the continuity equations [14-16]. For a complete description of semiconductor physical behavior, we also have to take into account the following equations:

$$
\operatorname{div}(\vec{D})=\rho-\rho_{\text {trap }}
$$




$$
\vec{E}=-\operatorname{grad} V
$$

Regarding the penultimate equation, $\rho$ is the space charge and $\rho_{\text {trap }}$ is the charge density contributed by traps and fixed charges. In the common acceptation, the electric field induction $\vec{D}=\varepsilon \vec{E}$, where $\vec{E}$ is the electric field and $\varepsilon$ is the electric permittivity of the material. In the last equation $V$ represents the electrostatic potential.

Replacing Eq. (2) in Eq. (1) and for a space charge $\rho$ specified as $\rho=q(p-n+N)$ with $N=N_{D}-N_{A}$ denoting the fully ionized net impurity distribution, we get a partial differential equation of elliptic type:

$$
\Delta V=-\frac{1}{\varepsilon}\left[q\left(p-n+N_{D}-N_{A}\right)+\rho_{\text {trap }}\right]
$$

where $N_{D}$ is the concentration of ionized donors and $N_{A}$ is the ionized acceptors concentration. Finally we can say that the electrostatic potential $V$ is the solution of the Poisson equation in (3).

\section{B. Physical simulations methodology}

Three-dimensional simulations of Hall cells were performed, with the aid of Synopsys Sentaurus TCAD software [17] that is able to solve the Poisson equation, both electrons and holes continuity equations.

The carrier transport process (electrostatic potential, current distributions) under magnetic field for semiconductor magnetic sensors with different geometries has been numerically modeled in three dimensions. Basically, at each point of the grid, three unknowns, namely $V, n, p$ are to be considered. Furthermore, three equations and the corresponding boundary conditions are required to solve the nonlinear system of partial differential equations. For the generation of a correct solution, a discretization of the Poisson's equation, electron and holes continuity equations is performed and used in conjunction with a coupled method. The latter, which is a generalization of the Newton method, is employed to compute the initial proposed system through a numerical iterative procedure.

The galvanic transport model used for carrier transport in magnetic fields allows for the correct consideration of the magnetic field acting on the semiconductor Hall structure. For a more detailed theoretical understanding of galvanomagnetic effects different books $[2,14]$ could be consulted. In order to analyze the effects of magnetic field in semiconductor structure, the transport equation of electrons and holes inside the device must be solved. By considering the magnetic fielddependent terms contributed by the effect of Lorentz force on the carriers, the usual drift-diffusion model of the carrier densities $\overrightarrow{J_{n}}$ and $\overrightarrow{J_{p}}$ are rewritten. Within its galvanic transport model, the Sentaurus software includes the effect of magnetic field on semiconductors, as follows:

$$
\begin{aligned}
\overrightarrow{J_{\alpha}}=\mu_{\alpha} \overrightarrow{g_{\alpha}}+\mu_{\alpha} & \frac{1}{1+\left(\mu_{\alpha}{ }^{*} B\right)^{2}}\left[\mu_{\alpha}{ }^{*} \vec{B} \times \overrightarrow{g_{\alpha}}+\mu_{\alpha}{ }^{*} \vec{B}\right. \\
& \left.\times\left(\mu_{\alpha}{ }^{*} \vec{B} \times \overrightarrow{g_{\alpha}}\right)\right]
\end{aligned}
$$

where $\alpha=n, p, \overrightarrow{g_{\alpha}}$ is the current vector without mobility and $\mu_{\alpha}{ }^{*}$ is the Hall mobility [17].

In this study, the mobility is doping dependent and the recombination processes are of the type Shockley-Read-Hall and Auger model as in [18]. The usual Dirichlet type boundary conditions prescribe the carrier concentrations and electrostatic potential at the contact region. The constructed SOI Hall device, simulated through three-dimensional physical simulations and analyzed, will be presented in details in Section IV.

\section{OPTIMUM HALL CELL MODEL}

A multitude of different Hall structures have been integrated in a non-fully depleted CMOS SOI technological process. This study focuses on a particular optimum structure, exhibiting good performance from both the offset and sensitivity point of views. Previous works of the authors have investigated the SOI XL Hall cell [8] and the SOI basic Hall cell [9], providing numerical estimations for their main parameters.

This structure has been called optimum due to the fact that it has the optimum shape and dimensions in terms of the performances envisaged to be achieved. The position of contacts with respect to borders is important in the offset analysis as contour errors might increase it. The optimum cell is a combination of increased size and contacts located at an optimum distance between the cells borders and the middle of the active region. Therefore, this structure has optimized dimension, for sensitivity maximization and offset reduction purpose.

The Optimum Hall cell, which is considered for detailed investigation, is modeled after the SOI fabrication process in which it had been fabricated. It is to be mentioned that the same Hall cell (but with an n-well) was also integrated in a regular CMOS technology (XFAB $0.35 \mu \mathrm{m}$, more precisely) and thoroughly tested by the author in several papers. The purpose is to make a comparison between the Hall cells fabricated in both regular bulk and SOI CMOS technological processes.

\section{A. Optimum Hall cell model based on SOI CMOS process}

This work focuses on the analysis of the Optimum Hall cell. Generally speaking, Hall cells are symmetrical and orthogonal structures. The three-dimensional model of the SOI Optimum Hall Cell is illustrated in Figure 1.

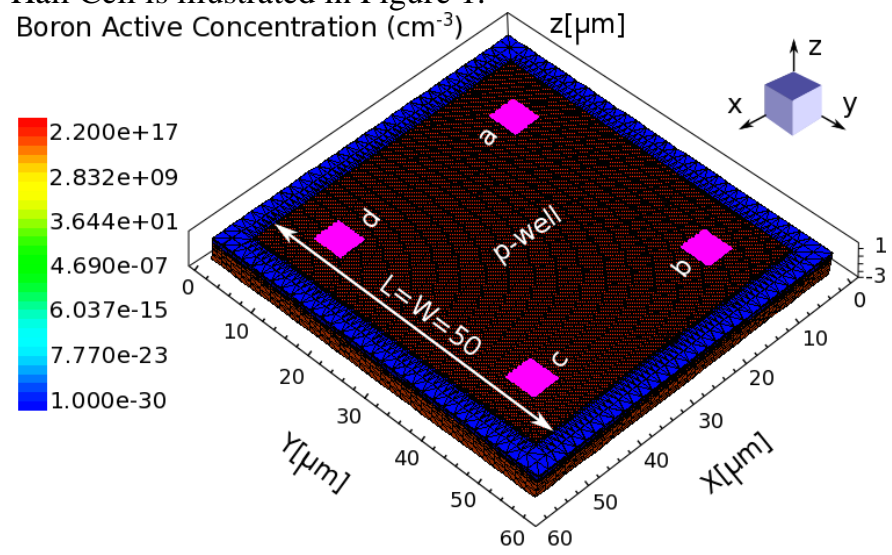

Figure 1. The three-dimensional model (meshed structure) of the SOI Optimum Hall cell

The color coded legend presents the doping concentration in the layers within the studied structure. The pink zones represent the four electrodes. The mesh is very fine as it has 
been adapted in order to provide accurate results, by introducing refinement windows at the contacts and at the interface between Silicon and $\mathrm{SiO}_{2}$. Moreover, the geometrical design features of the structure are included in Table I.

TABLE I. SOI OPTIMUM HALL CELL DESIGN FEATURES

\begin{tabular}{|c|c|}
\hline Parameter & Value \\
\hline$L(\mu \mathrm{m})$ & 50 \\
\hline$W(\mu \mathrm{m})$ & 50 \\
\hline Contacts dimension $s(\mu \mathrm{m})$ & 4.7 \\
\hline
\end{tabular}

The doping concentrations used in the modeling of the Hall cell, by close reproduction of a XFAB SOI XI10 integration process are to be noted. Therefore, Figure 2 depicts the doping profiles of the layers used in the simulation of the SOI Optimum Hall cell. The Oy cut represents the cut of the three-dimensional structure with a plane parallel with the $x \mathrm{O} z$ plane. The handle substrate was modeled at $3 \mu \mathrm{m}$, as it was sufficient for the present simulations needs.

$\mathrm{z}[\mu \mathrm{m}] \uparrow$

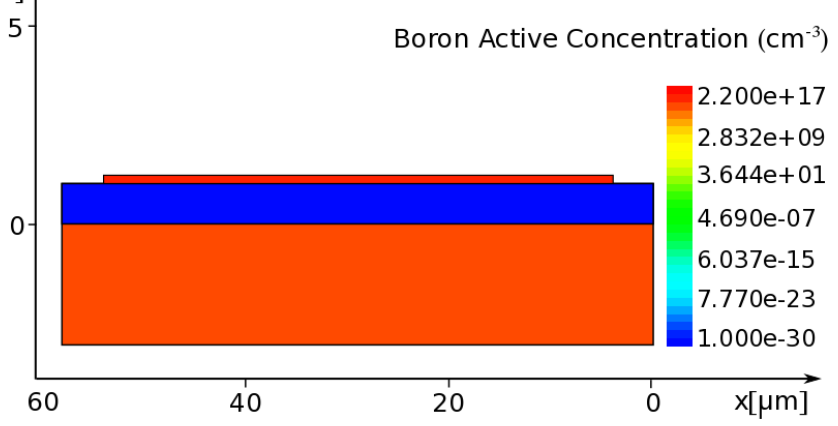

Figure 2. Oy Cut through the SOI Optimum Hall cell, depicting the doping profiles

Figure 3 presents the stacking of the layers, according to a SOI XFAB XI10 fabrication process. This means that the active silicon layer is found on top of the dielectric buried silicon oxide $\left(\mathrm{SiO}_{2}\right)$ layer, which is in its turn found on the silicon substrate, or handle wafer.

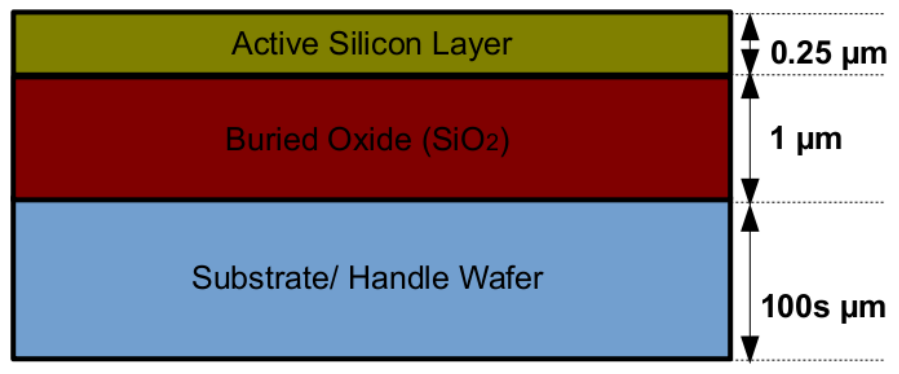

Figure 3. The fabrication layers of an SOI XFAB XI10 integration process

Table II shows more precisely the doping profiles used in the simulation of the SOI Optimum Hall cell. We have to mention that all the doping profiles in Table II are defined as constant doping profiles. The thicknesses of the layers used in the SOI Hall cell model are all considered according to the details in Figure 3, with the addition that the depth of the implantation profile in the p-well occupies the whole active Silicon, namely $250 \mathrm{~nm}$.
The depth of the active silicon layer in SOI integration process is smaller than in a regular CMOS. By consequence, an increased sensitivity and increased input resistance are expected. Also, a smaller biasing current could be used and the power consumption could be diminished.

TABLE II.

DOPING CONCENTRATIONS IN THE SOI HALL CELL MODEL

\begin{tabular}{|c|c|c|}
\hline Layer & Type & Numerical value \\
\hline $\begin{array}{c}\text { Wafer (handle) } \\
\text { substrate }\end{array}$ & $\begin{array}{c}\mathrm{Si}, \mathrm{p} \text {-doped } \\
\text { (Boron) }\end{array}$ & $6.5 \mathrm{E}+14 \mathrm{~cm}^{-3}$ \\
\hline Dielectric & Buried Silicon Oxide, $\mathrm{SiO}_{2}$ \\
\hline $\begin{array}{c}\text { p-well in active } \\
\text { Silicon layer }\end{array}$ & $\begin{array}{c}\mathrm{Si}, \mathrm{p} \text {-doped } \\
\text { (Boron) }\end{array}$ & $2.2 \mathrm{E}+17 \mathrm{~cm}^{-3}$ \\
\hline
\end{tabular}

\section{B. Hall cell biasing and electrostatic potential}

Simulations were performed in TCAD Sentaurus Synopsys, with the purpose to analyze the behaviour of the SOI Optimum Hall cell. The considered structure was both simulated without magnetic field, producing an information on the offset and with magnetic field $(B=0.5 \mathrm{~T})$, producing a result on the Hall voltage respectively.

The Hall device has four electric contacts (denoted from $a$ to $d$ ), amongst which two are used for biasing and the other two for sensing. More specifically, if the device is biased with voltage on the electrode $a$, this will force a current to flow between $a$ and $c$ contacts, as in Figure 4. The Hall voltage is recorded between the opposite contacts ( $b$ and $d$ in this case).

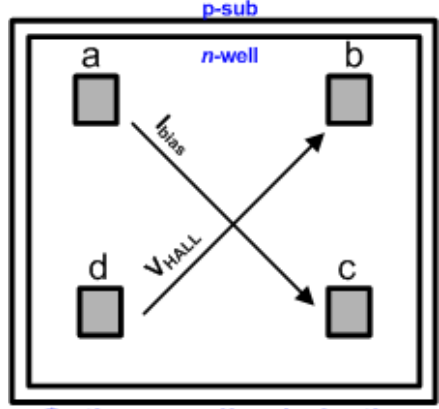

\begin{tabular}{|c|c|c|}
\hline \multirow{2}{*}{ Phases } & \multicolumn{2}{|c|}{ Biasing/sensing } \\
\cline { 2 - 3 } & $I_{\text {bias }}$ & $V_{\text {hall }}$ \\
\hline $\mathrm{P}_{1}$ & $a$ to $c$ & $b$ to $d$ \\
\hline $\mathrm{P}_{2}$ & $d$ to $b$ & $a$ to $c$ \\
\hline $\mathrm{P}_{3}$ & $c$ to $a$ & $d$ to $b$ \\
\hline $\mathrm{P}_{4}$ & $b$ to $d$ & $c$ to $a$ \\
\hline
\end{tabular}

Optimum cell polarization

Figure 4. Optimum cell biasing scheme (Phase 1) (left-hand side) and the four biasing phases (right-hand side)

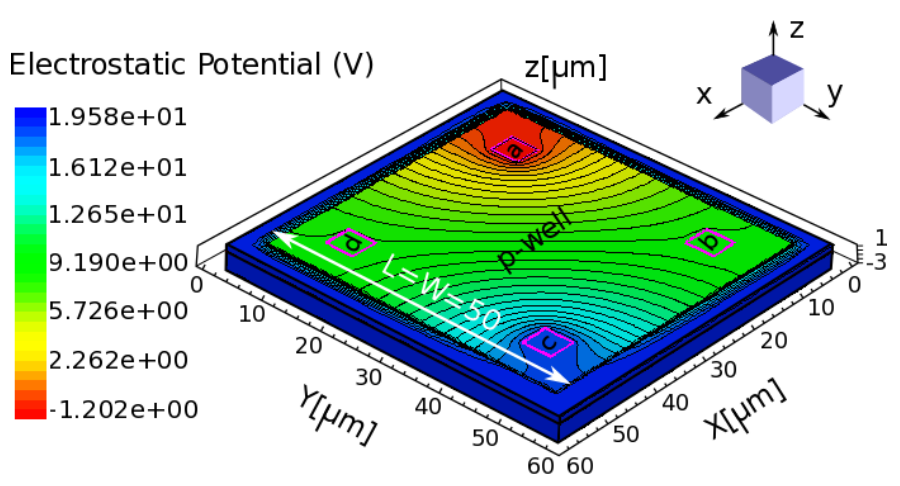

Figure 5. Electrostatic potential distribution of the SOI Optimum Hall cell

Figure 5 presents the electrostatic potential distribution of 
the SOI Optimum Hall cell, where the device has been biased with voltage bias up to $20 \mathrm{~V}$ on the "red" contact, and placed under a magnetic field of magnetic induction $\mathrm{B}=0.5 \mathrm{~T}$. As we know, in the presence of magnetic field, the electric carriers deviate under the influence of the Lorentz force and there is a non-zero voltage difference between the two opposite contacts.

\section{RESULTS AND DISCUSSION}

The present work is focused on a particular Hall geometry in a CMOS SOI technology. The Optimum Hall cell has been integrated in an XFAB SOI XI10 process technology, and thoroughly tested. Before proceeding to devices integration, the three-dimensional physical simulations are important tool for the designer as they can give a numerical estimation of the important characteristic parameters and provide a performance evaluation.

This section presents the results on the main parameters for the Optimum Hall cell in SOI technology. By building the three dimensional model of this structure and using the set of equations governing its behaviour, we could have a numerical estimation of the input resistance, electrostatic potential, space charge distribution, Hall voltage, absolute sensitivity, currentvoltage characteristic, temperature variation.

To validate the simulations, experimental results have been used. Around a dozen different samples of the same SOI Optimum Hall cell, from two different wafers, have been tested.

\section{A. Input resistance and absolute sensitivity evaluation}

Regarding a sensor's performance, an important figure of merit is its sensitivity. The absolute sensitivity $S_{A}$ of a Hall magnetic sensor is given by the ratio between the Hall voltage $V_{H A L L}$ and the magnetic field induction $B$ [19]:

$$
S_{A}=\left|\frac{V_{H A L L}}{B}\right|=\frac{G r_{H}}{n q t} I_{\text {bias }}
$$

where $G$ is the geometrical correction factor, $I_{\text {bias }}$ is the biasing current, $r_{H}$ is the Hall scattering factor, $n$ is the carrier density and $t$ is the thickness of the active region [19]. One could note at this point that in order to increase the sensitivity of a Hall sensor, either a lower doping concentration $n$ in the active region or a smaller depth $t$ of the active profile should be used.

At this point, results (both measured and simulated data) regarding important parameters of the Optimum Hall cell are introduced. Figure 6 depicts the current-voltage characteristic (both simulated and measurement data) of the SOI Optimum Hall cell. The measured input resistance is computed as the inverse of the slope of the regression line drawn through the points in Figure 7 and it equals to $\mathrm{R}=6.47 \mathrm{k} \Omega$.

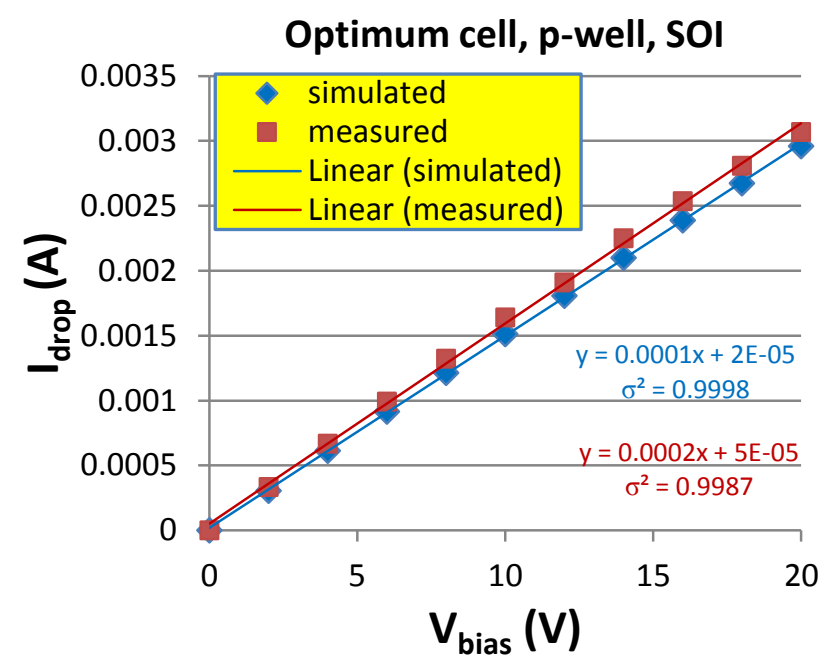

Figure 6. I-V characteristic of the SOI Optimum Hall cell, (simulated and measured data)

In Figure 7 the absolute sensitivity at room temperature (both simulated and measured data) versus the biasing current is presented for the SOI Optimum Hall cell. The simulated data was obtained at $\mathrm{T}=300 \mathrm{~K}$ and $\mathrm{B}=0.5 \mathrm{~T}$.

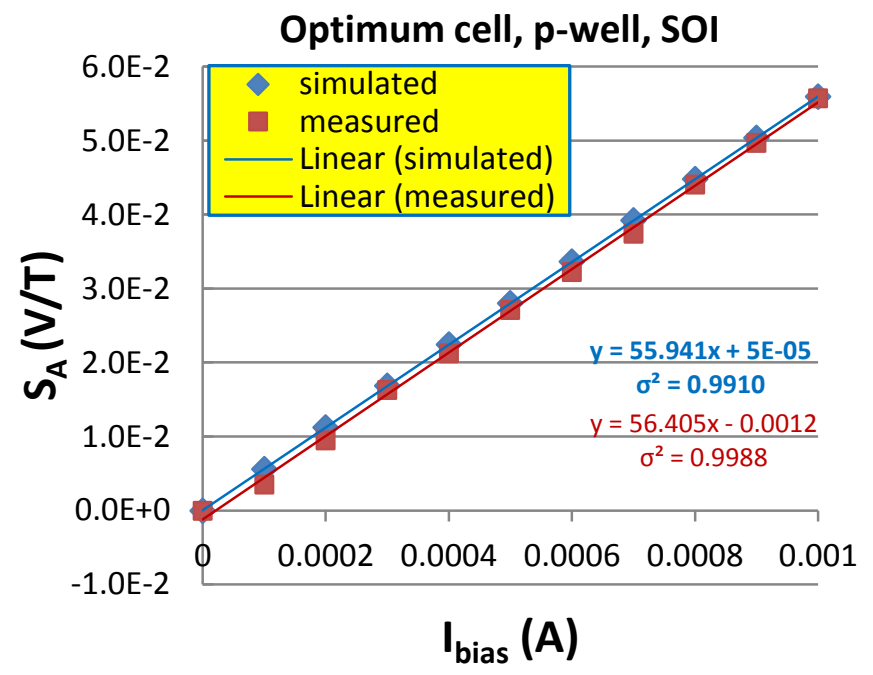

Figure 7. Absolute sensitivity vs. biasing current for the SOI Optimum Hall cell (measured and simulated data)

\section{B. SOI Optimum Hall cell temperature behavior investigation}

The effect of temperature on the Optimum SOI Hall cell behaviour (input resistance, absolute sensitivity, etc.) was experimentally measured. A $20-120{ }^{\circ} \mathrm{C}$ temperature range was considered, using a Thermotron oven (model S1.2CE).

Figure 8 presents the input resistance temperature variation, for the SOI Optimum Hall cell. 


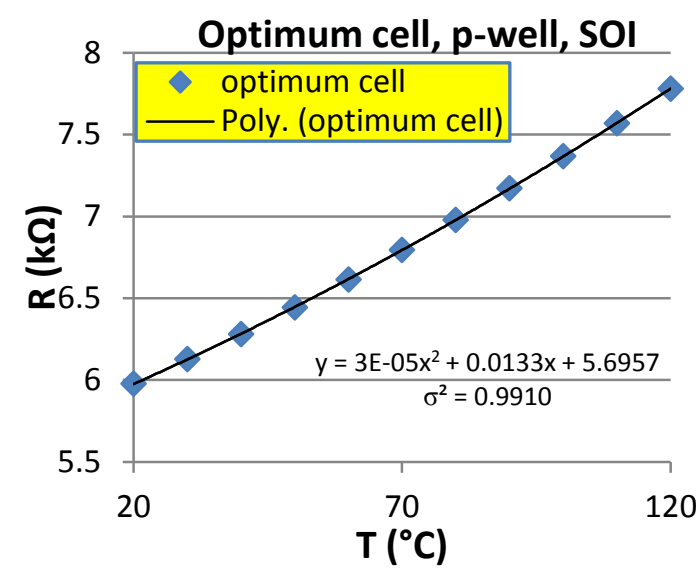

Figure 8. Measured input resistance $v s$. temperature

Previous studies have been carried by the first author to investigate the temperature behaviour of the sensitivity of the regular bulk CMOS Hall cells [20], particularly taking into account the freeze-out effect. In this work [20], it has been observed and demonstrated analytically the quadratic behaviour of the current-related sensitivity with the temperature.

The temperature dependence of the absolute sensitivity with the temperature obeys the following equation, in which the variation of the parameters with temperature has been taken into account.

$$
S_{A}(T)=G \frac{r_{H}(T)}{n(T) q t_{e f f}(T)} I_{\text {bias }}
$$

The temperature dependence of the magnetic sensitivity of the Hall cells is influenced by the temperature variation of two competing factors namely the freeze-out effect of the electron density and the Hall scattering coefficient respectively. As it has been investigated in details in [20], this produces a second order effect of the absolute sensitivity variation with the temperature.

In order to identify the temperature variation of the absolute sensitivity for the SOI Optimum Hall cell, corresponding measurements have been performed.

Figure 9 illustrates the absolute sensitivity plotted against the temperature, for $I_{\text {bias }}=1 \mathrm{~mA}$.

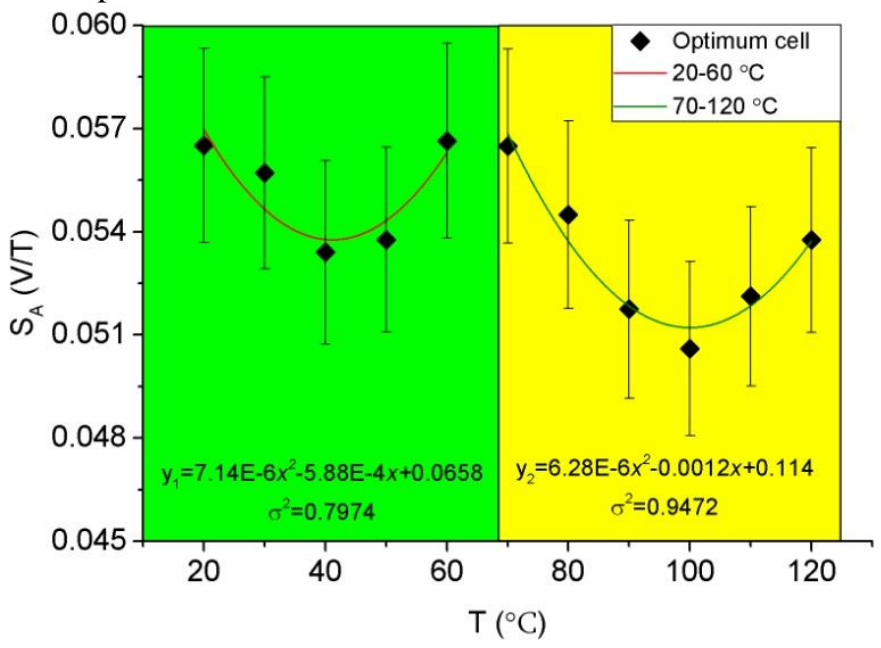

Figure 9. Measured absolute sensitivity $v s$. temperature
By examining the data in Figure 9, the total temperature drift value of the absolute sensitivity, in the considered interval, is $-2.74 \mu \mathrm{V} / \mathrm{TK}$. The experimental curves on both considered intervals $\left(20-60{ }^{\circ} \mathrm{C}\right.$ and $\left.70-120{ }^{\circ} \mathrm{C}\right)$ have been fitted with second order polynomials, and the errors bars corresponding to $\pm 3 \sigma$ have been included.

We can notice that in the temperature range $20-60{ }^{\circ} \mathrm{C}$, a quadratic behaviour of the absolute sensitivity with the temperature is obtained, with a minimum around $40{ }^{\circ} \mathrm{C}$. Even though $\sigma^{2}=0.79$ for this fitting, our intention was more to emphasize the general tendency of the sensitivity temperature dependence. Moreover, in the interval $70-120{ }^{\circ} \mathrm{C}$, there is a more abrupt decrease of the absolute sensitivity with the temperature, however still a second order polynomial dependence, with a minimum observed around $100{ }^{\circ} \mathrm{C}$.

Further on, Figure 10 presents the absolute sensitivity temperature dependence, for different biasing currents $\left(I_{\text {bias }}=0.3 \mathrm{~mA}, I_{\text {bias }}=0.5 \mathrm{~mA}, I_{\text {bias }}=0.7 \mathrm{~mA}\right.$ and $\left.I_{\text {bias }}=1 \mathrm{~mA}\right)$.

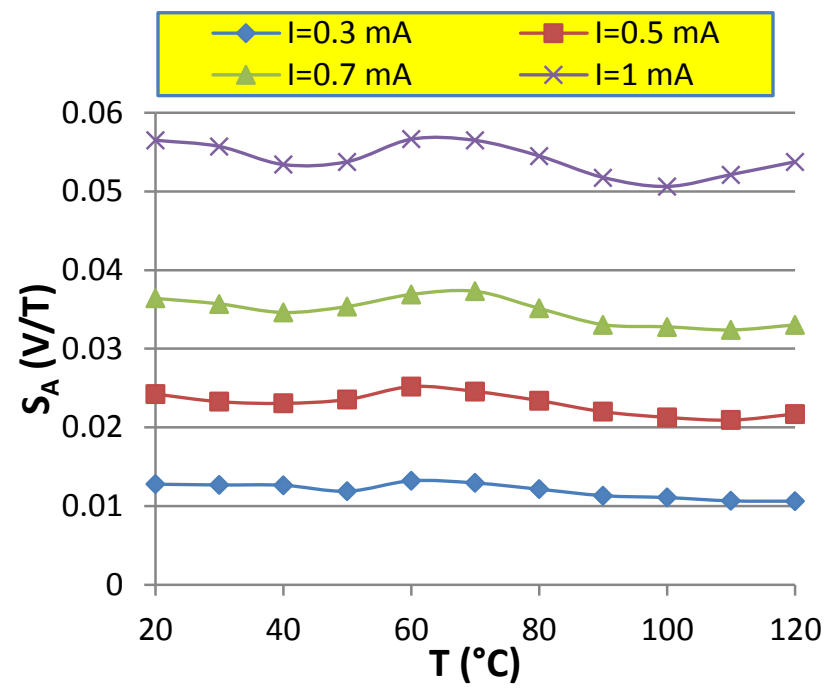

Figure 10. Measured absolute sensitivity $v s$. temperature, for different biasing currents

\section{Hall cells offset measurement}

It is well known that the offset (recorded for no magnetic field) is a parasitic voltage and it is obviously our aim to reduce it as much as we can. From the electronics point of view, the residual offset is obtained as an average on several phases, by making use of the current-spinning technique:

$$
V_{\text {offset, residual }}=\frac{1}{n} \sum_{i=1}^{n}(-1)^{i+1} V_{i}
$$

where $V_{i}$ is the individual offset of each phase, measured in $\mathrm{V}$, and $n$ is the total number of phases. The residual offset can be obtained as a result of 2-phase or 4-phase averaging. In the case of four phase current spinning, the considered phases sequence is $+V_{1},-V_{2},+V_{3},-V_{4}$. The unit of $V_{\text {offset,residual }}$ is obviously V.

Figures 11 and 12 present the 4-phase residual offset, measured for the SOI Optimum Hall cell, for both current and voltage bias. Even though there is a direct relation between the voltage and current through the resistance, the offset measured for voltage biasing cannot be linked to the offset measured for 
current biasing through the resistance, due to the randomness of the offset generation itself. Investigation in the offset behavior with both types of polarization is necessary and beneficial to the circuit designer [19].

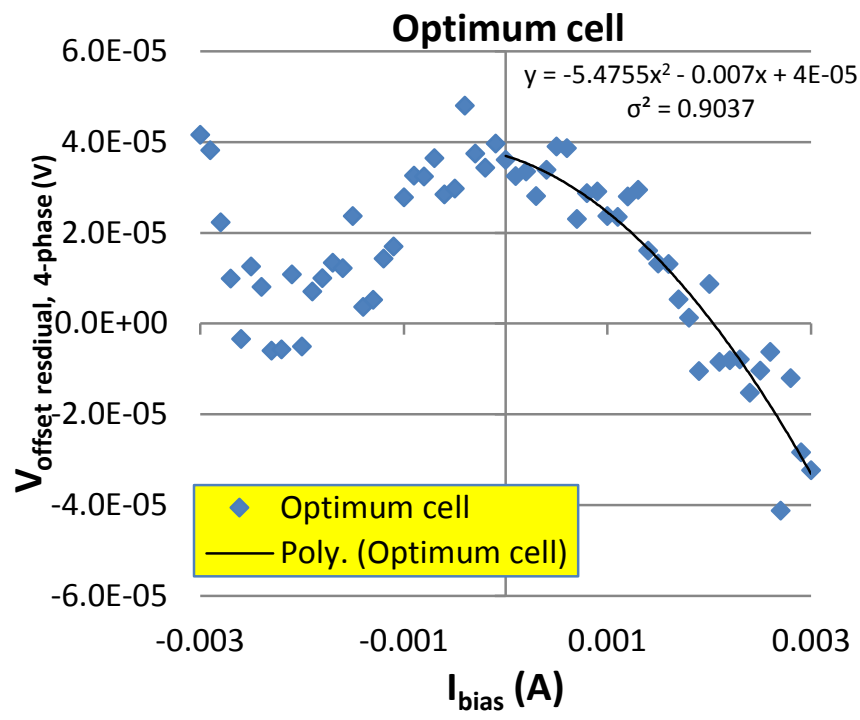

Figure 11. Measured four-phase residual offset voltage $v s$. biasing current, for $I_{\text {bias }}=0-3 \mathrm{~mA}$

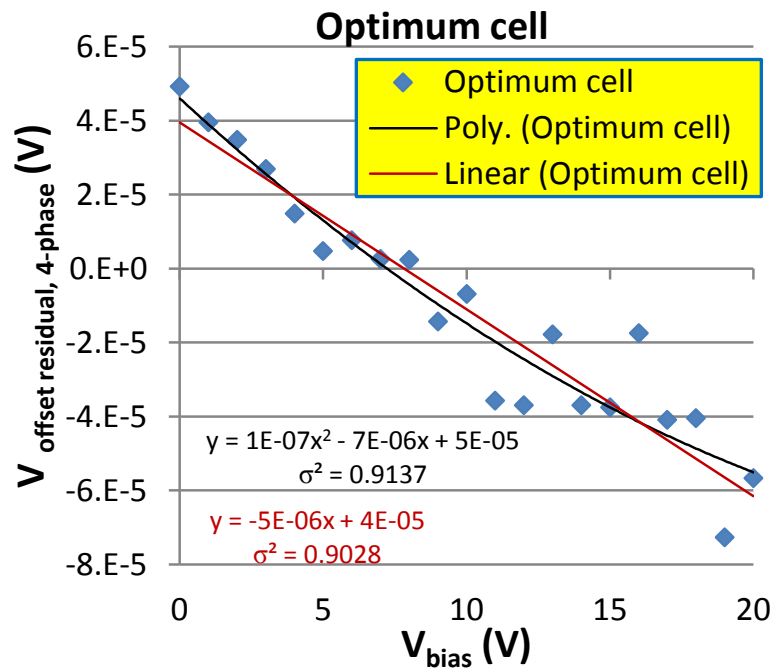

Figure 12. Measured four-phase residual offset voltage $v s$. biasing voltage, for $V_{\text {bias }}=0-20 \mathrm{~V}$

The curve in Figure 11 has been fitted with a second order polynomial, in the interval of positive biasing current voltage from 0 to $3 \mathrm{~mA}$. This is in accordance with the quadratric dependence of the 4-phase residual offset voltage with the biasing current proven in [21]. The curve in Figure 12 has been fitted with both a linear and second order polynomial respectively. We can see that on one hand the residual 4-phase offset values do not exceed $40 \mu \mathrm{T}$ for current biasing of up to $3 \mathrm{~mA}$, and on the other hand, the residual offset does not exceed $60 \mu \mathrm{T}$, for voltage biasing of up to $20 \mathrm{~V}$.

\section{Hall mobility investigation}

The model used in Sentaurus, for the doping-dependent mobility calculation in silicon [17] relies on the Masetti formula [22]:

$$
\begin{gathered}
\mu_{\text {dop }}=\mu_{\min 1} e^{-\frac{P_{C}}{N_{A, 0}+N_{D, 0}}}+\frac{\mu_{\text {const }}-\mu_{\text {min } 2}}{1+\left(\frac{N_{A, 0}+N_{D, 0}}{C_{r}}\right)^{\alpha}} \\
-\frac{\mu_{1}}{1+\left(\frac{C_{s}}{N_{A, 0}+N_{D, 0}}\right)^{\beta}}
\end{gathered}
$$

where $\mu_{1}, \mu_{\min 1}$ and $\mu_{\min 2}$ are reference mobilities, $C_{s}, C_{r}$ and $P_{C}$ are the reference doping concentrations. Numerical values of these parameters enumerated above, can be found in [17]. Additionally $\alpha$ and $\beta$ are two coefficients, specific for silicon, whose values are $\alpha=0.680$ (for electrons) and $\alpha=0.719$ (for holes) and $\beta=2$ (for both holes and electrons).

Also, it is worth mentioning that the low doping reference mobility $\mu_{\text {const }}$ is calculated according to the constant mobility model in Sentaurus, which accounts only for phonon scattering and by consequence it is dependent only on the lattice temperature.

This section presents considerations on the Hall mobility, as was extracted through simulations on the SOI Optimum Hall cell. With respect to the studied structure, the Hall mobility presented in Figure 13 corresponds to the holes $\mu_{p}{ }^{*}$, as it was introduced in Eq. (8).

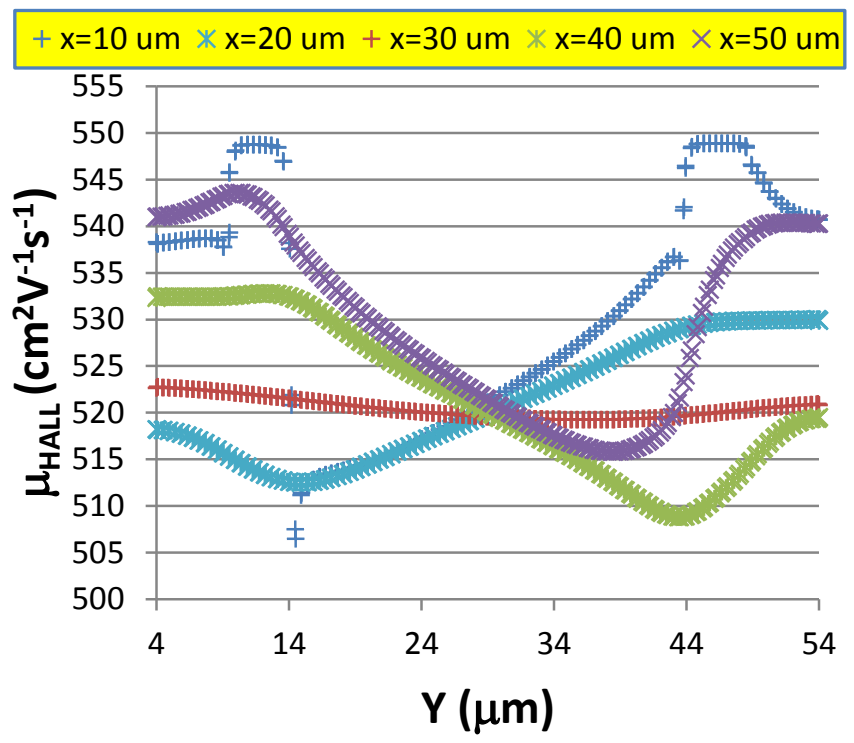

Figure 13. Simulated Hall mobility, for different 0x cuts

The graph above presents the Hall mobility, versus Oy axis, for different $\mathrm{x}$ cuts, for $V_{\text {bias }}=5 \mathrm{~V}$. This simulated data has been obtained at the very surface of the device, as preliminary to this the considered Hall structure has been cut with a plane parallel to $z=1.25 \mu \mathrm{m}$. The data, which is the result of the three-dimensional simulations, has been taken for $\mathrm{T}=300 \mathrm{~K}$. We can see a maximum of $\mu_{H A L L}=550 \mathrm{~cm}^{-2} \mathrm{~V}^{-1} \mathrm{~s}^{-1}$.

E. Main parameters summary of the Optimum Hall cell in both SOI and regular bulk CMOS technological processes

When deciding on the choice of the technology, the performance to be achieved (high sensitivity, lower offset, lower offset temperature drift, lower power consumption, etc.) and the biasing conditions are important considerations to be taken into account.

Table III summarizes the SOI Optimum Hall cell 
characteristic parameters. The presented results are measurement data, obtained at room temperature. The currentrelated sensitivity $S_{I}=\frac{S_{A}}{I_{\text {bias }}}$ and voltage-related sensitivity $S_{v}=\frac{S_{A}}{V_{\text {bias }}}$ are also included.

In order to make a comparative analysis of the two technological processes, table IV presents the bulk CMOS optimum Hall cell main parameters, obtained through measurements at room temperature and magnetic field $\mathrm{B}=0.5 \mathrm{~T}$. It is to be mentioned at this point that we are presenting here the results of an n-well active region regular bulk CMOS Optimum Hall structure, but the geometry is the same.

TABLE III

\begin{tabular}{|c|c|}
\hline Parameters & SOI device \\
\hline$R_{\text {input }}(\mathrm{k} \Omega)$ & 6.47 \\
\hline$S_{I}(\mathrm{~V} / \mathrm{AT})$ & 55 \\
\hline$S_{V}\left(\mathrm{~T}^{-1}\right)$ & 0.0085 \\
\hline$S_{A}$ temperature drift $(\mu \mathrm{V} / \mathrm{TK}), I_{\text {bias }}=1 \mathrm{~mA}$ & -2.74 \\
\hline Max offset level $(\mu \mathrm{T}), I_{\text {bias }}=0-3 \mathrm{~mA}$ & 40 \\
\hline Max offset level $(\mu \mathrm{T}), V_{\text {bias }}=0-20 \mathrm{~V}$ & 60 \\
\hline
\end{tabular}

TABLE IV. BULK CMOS OPTIMUM HALL CELL MAIN PARAMETERS

\begin{tabular}{|c|c|}
\hline Parameters & Bulk device \\
\hline$R_{\text {input }}(\mathrm{k} \Omega)$ & 1.8 \\
\hline$S_{A}(\mathrm{~V} / \mathrm{T}), I_{\text {bias }}=0.5 \mathrm{~mA}$ & 0.032 \\
\hline Offset temperature drift, $\left(\mu \mathrm{T} /{ }^{\circ} \mathrm{C}\right)$ & 0.328 \\
\hline
\end{tabular}

In [4] it has been proposed an increase of the SOI Hall cells sensitivity by reaching $S_{I}=100 \mathrm{~V} / \mathrm{AT}$, with a p-type Silicon as active device layer. However, there is no mention of the offset levels or input resistance. Our paper offers a detailed analysis of the offset and biasing conditions. In [23], extensive experimental results for the resistance of SOI for different biasing currents and various temperatures are provided.

\section{CONCLUSION AND FUTURE PERSPECTIVES}

A multitude of Hall cells have been integrated in a CMOS SOI $1 \mu \mathrm{m}$ non-fully depleted technological process. In particular, this paper was intended to analyze the behaviour of the Optimum Hall cell in a SOI fabrication process, by building a three-dimensional physical model of the structure and performing simulations.

To assess the performance of Hall cells, the characteristic parameters have been extracted. To this aim, the Hall voltage, absolute sensitivity and input resistance of the Optimum SOI Hall cell have been both extracted through simulations and measured. The electrostatic potential distribution and space charge have also been analyzed. Experimental results have been employed to validate the simulations results.

Temperature investigation into the behaviour of the absolute sensitivity has been also part of the focus of this study. Moreover, the work was also intended to characterize the offset of the considered Hall cell. To this purpose, the dependence of the residual offset with both biasing current and biasing voltage has been measured. Additionally, a Hall mobility investigation onto the considered device was made using three-dimensional physical simulations.

As next objective, all SOI Hall cells will be measured, using electrical and magnetic testing. We will be further using the obtained experimental results on the studied devices to harmonize the three-dimensional physical model developed.

\section{ACKNOWLEDGMENT}

The first author, Maria-Alexandra Paun, wishes to thank the Swiss National Science Foundation (SNSF) from Switzerland for the promotion and encouragement of the scientific research of young doctors, respectively by providing the funding for her advanced postdoctoral fellowship at Cambridge University, UK and the return phase at EPFL, Switzerland.

\section{REFERENCES}

[1] Popovic, R.S., "Hall Effect Devices: Magnetic Sensors and Characterization of Semiconductors", Adam Hilger, 1991.

[2] Ramsden, E., "Hall-Effect Sensors - Theory and Applications", (Second Edition), Elsevier, 2006.

[3] Dolgyi, L. N., Lovshenko, I. Yu., Nelayev, V. V., "Technology and Electric Characteristics of the Field-Effect Hall Sensor Based on SOI Structure”, Russian Microelectronics, Vol. 42, No. 7, pp. 368-372, 2013.

[4] Singh N., Estrada H. V., “A high sensitivity Hall sensor fabricated on a SOI wafer using surface micromachining technique”, Micromachining and Microfabrication Process Technology XIV, edited by M.-A. Maher, J.-C. Chiao, P. J. Resnick, Proceedings of SPIE, Vol. 7204, 720406, 2009, pp 1-8.

[5] Blagojevic, M., Kayal, M., De Venuto, D., "FD SOI Hall sensor electronics interfaces for energy measurement", 6th International Workshop on Epitaxial Semiconductors on Patterned Substrates and Novel Index Surfaces (ESPS-NIS 2006), Nottingham, England, April 03-05, 2006, Microlectronics Journal, Vol. 37, Issue 12, Special Issue, pp. 1576-1583, 2006.

[6] Blagojevic, M., De Venuto, D., Kayal, M., "SOI Hall sensor based solid state meter for power and energy measurements”, edited by Rocha, D., Sarro, P.M., Vellekoop, M.J., Proceedings of the IEEE Sensors 2004 Conference, Vienna, Austria, October 24-27, 2004, Vols. 1-3, Book Series: IEEE Sensors, pp. 1040-1043, 2004.

[7] Portmann, L., Ballan, H., Declercq M., "A SOI CMOS Hall effect sensor artchitecture for high temperature applications (up to $300^{\circ} \mathrm{C}$ ) ', IEEE, 2002, pp. 1401-1406.

[8] Paun, M.A.., "Three-dimensional simulations in optimal performance trial between two types of Hall sensors fabrication technologies", Journal of Magnetism and Magnetic Materials, Vol. 391, pp. 128-128, 2015.

[9] Paun, M.A., Udrea, F., "SOI Hall Cells Design Selection using ThreeDimensional Physical Simulations", Journal of Magnetism and Magnetic Materials, Vol. 372, pp. 141-146, 2014.

[10] Paun, M.A., Sallese, J.M., Kayal, M., "Comparative Study on the Performance of Five Different Hall Effect Devices", Sensors, ISSN 1424-8220, Vol. 13, Issue 2, 2013, pp. 2093-2112.

[11] Paun, M.A., Sallese, J.M., Kayal, M., “Offset And Drift Analysis Of The Hall Effect Sensors. The Geometrical Parameters Influence”, Digest Journal of Of Nanomaterials And Biostructures, Vol. 7, Issue 3, pp. 883891, 2012.

[12] Paun, M.A., Sallese, J.M., Kayal, M., "Geometry influence on the Hall effect devices performance", U.P.B. Scientific Bulletin, Series A, Vol. 72, Issue 4, 2010. 
[13] Paun, M.A., Sallese, J.M., Kayal, M., “A Circuit Model for CMOS Hall Cells Performance Evaluation including Temperature Effects", Advances in Condensed Matter Physics, Article Number: 968647, 2013.

[14] Sze, S. M., Ng, K. K., "Physics of semiconductor devices", Third Edition, John Wiley and Sons, 2007.

[15] Selberherr, I. S., "Analysis and Simulation of Semiconductor Device”, Vienna, Austria, Springer-Verlag, 1984.

[16] Allegretto, W., Nathan, A., Baltes, H., "Numerical Analysis of Magnetic-Field-Sensitive Bipolar Devices", IEEE Transactions On Computer-Aided Design, Vol. 10, No. 4, 1991.

[17] Synopsys TCAD software tools online documentation, access at the address: http://www.synopsys.com/Tools/TCAD

[18] Jovanovic, E., Pesic, T., Pantic, D., "3D Simulation of Cross-shaped Hall Sensor and Its Equivalent Circuit Model", Proceedings of the $24^{\text {th }}$ International Conference on Microelectronics (MIEL 2004), Vol. 1, pp. 235-238, 16-19 May, 2004.
[19] Popovic, R.S, "Hall Effect Sensors", second edition, Institute of Publishing Bristol and Philadelphia, 2004.

[20] Paun, M.A., Sallese, J.M., Kayal, M., "Temperature considerations on Hall Effect sensors current-related sensitivity behaviour", Analog Integrated Circuits And Signal Processing, Vol. 77, Issue 3, Special Issue: SI, pp. 355-364, 2013.

[21] Ruther, P., Schiller, U., Buesser, W., Janke, R. Paul, O., "Thermomagnetic Residual Offset in Integrated Hall plates", Proceedings of the $1_{\text {st }}$ IEEE Sensors Conference, Orlando, FL, USA, June 12-14, pp. 763-766, 2002.

[22] Masetti, G., Severi, M., Solmi, S., "Modeling of Carrier Mobility Against Carrier Concentration in Arsenic-, Phosphorus-, and BoronDoped Silicon," IEEE Transactions on Electron Devices, Vol. ED-30, No. 7, pp. 764-769, 1983.

[23] Chen, C., Huang, J. S., Liuao, C. N., Tu, K. N., "Dopant activation of heavily doped silicon-on-insulator by high density currents", Journal of Applied Physics, Vol. 86, No. 3, pp. 1552-1557, 1999. 